\title{
Electron Precession for Routine Crystallography
}

\author{
Christopher S. Own*, Laurence D. Marks* \\ * Dept. of Materials Science and Engineering, Northwestern University, 2220 Campus Dr., Cook \\ 2036, Evanston, IL 60208
}

Transmission electron microscopy is a powerful tool in crystallography, offering direct atomic imaging and acquisition of chemical and spatial information, while allowing simultaneous acquisition of structural information in reciprocal space to rather high resolution. However, while it has been used uniquely to solve many structural materials problems, as a standalone crystallography tool, TEM is still hindered by the dynamical scattering that prevents straightforward interpretation of intensity data in the majority of experimental conditions. This has led to complex schemes involving recovery of exact experimental variables or application of prior knowledge of the structure in order to solve some materials problems [1-2]. Many modern nanomaterials problems are now tractable only with electron microscopy; overcoming what is fundamentally a data quality problem will further enhance the TEM's efficiency and utility in many areas.

Advances such as Z-contrast imaging enhance the interpretability of image data through clever experiment geometry but a practical analog in diffraction, which would be useful in experimental structure studies, has been elusive. The precession technique has been shown to improve the quality of electron diffraction data to the point where it can be used in some cases completely straightforwardly in the direct solution of complex structures [3-6]. This has considerable ramifications for many materials problems in the field where it can enhance usefulness of diffraction not only for direct structure solution from intensities but also for potentially improving other techniques such as phase extension of high resolution CTEM images and structure completion.

We have implemented three precession systems in a variety of conventional TEMs and have investigated the behavior of datasets taken under various conditions by comparison with multislice simulation [6-8]. Precession datasets captured under ideal conditions appear to be much less sensitive to thickness than conventional diffraction datasets and indeed exhibit a pseudo-kinematical nature. However, their correct interpretation is still a dynamical one and thickness effects, while slight, follow a new trend that has not been seen previously in electron diffraction (see Figure 1). A correction of the data depending on approximate thickness, discussed below, is still preferred in order to directly interpret the intensities in a kinematical capacity.

The quality of precession data is highly dependent upon optimization of certain experiment parameters. Due to some inherent insensitivity to specimen thickness, the shape constraint is less limiting than in conventional electron diffraction (see Figure 2 for comparison). For example, data from powder specimens that do not cleave well (wherein thickness averaging is largely unavoidable) appear to be very amenable for precession crystallography studies. However, optical constraints become far more critical in precession: beam alignment with the optical axis, specimen alignment with optimal objective excitation, and distortion compensations must be carefully adjusted to ensure near-ideal data collection conditions. While conventional electron diffraction has traditionally been free from concerns of optical aberrations, the domain of aberration correction offers considerable advantages for precession diffraction. With a corrector in the column, enhanced precession 
conditions - namely fine probe localization and large cone semi-angle - are more easily met. An implementation on a corrected instrument will be presented showing the inherent advantages and ease of characterization when precession is combined with aberration correction.

Finally, we have examined the validity of the only available theoretical model of precession, based upon two-beam theory [9]. In the systems investigated, we have found little support for its effectiveness in either real or simulated data. A more accurate model would be useful for the correction of intensities for thickness and cone semi-angle; a theoretical model of precession based upon a geometrical reduction of channeling conditions will be presented.

\section{References:}

[1] M.A. O'Keefe, et al.Mat. Res. Soc. Symp. Proc. 159 (1989) 453-458.

[2] W. Sinkler and L.D. Marks, Ultramicroscopy. 75 (1999) 251-268.

[3] R. Vincent and P.A. Midgley, Ultramicroscopy. 53 (1994) 271-282.

[4] M Gemmi, et al., Acta Cryst. Sect. A. A59 (2003) 117-126.

[5] J. Gjønnes, et al., Microsc. Microanal. 10 (2004) 16-20.

[6] C.S. Own, et al., Acta Cryst. Sect. A. (submitted).

[7] C.S. Own, et al., Microsc. Micronal. 10 (2004) 96-104.

[8] C.S. Own, et al., Rev. Sci. Instr. (in press).

[9] K. Gjønnes, Ultramicroscopy. 69 (1997) 1-11.

[10] This work possible with support from UOP, STCS, DOE (DE-FG02-03ER 15457), and the Fannie and John Hertz Foundation.
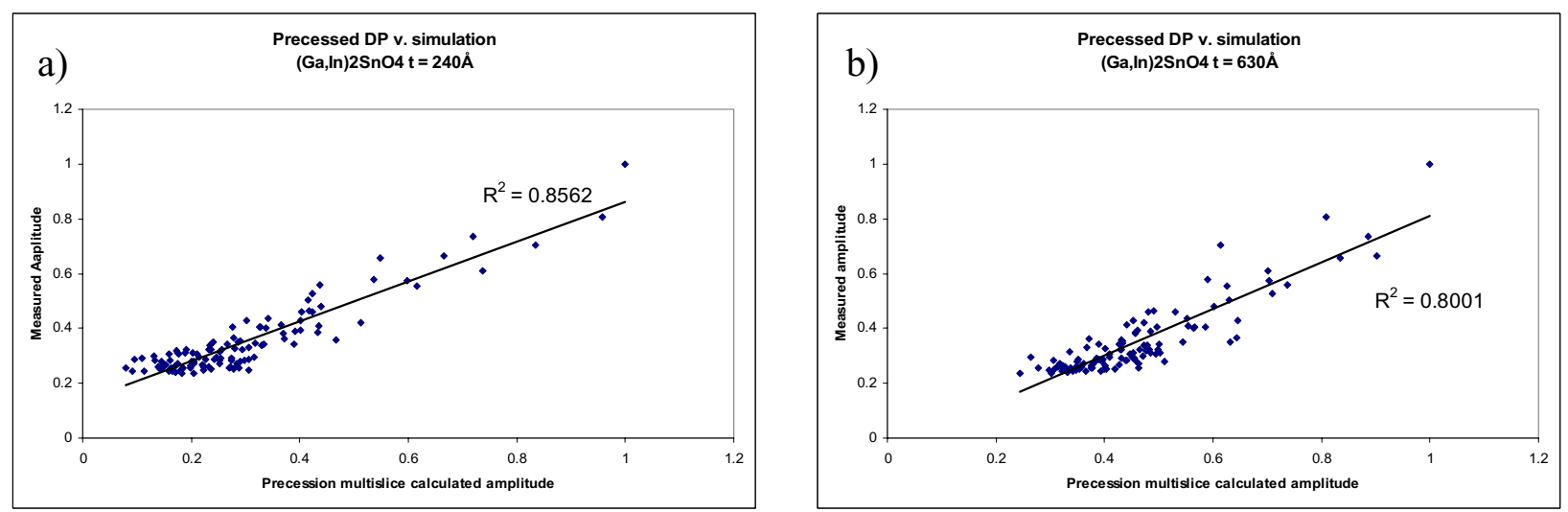

Fig. 1. Experimental precession data v. simulation for (a) $t=240 \AA$ and (b) $t=630 \AA$, arbitrary units. $\alpha=24 \mathrm{mrad}$. The data matches simulation closely and the linearity is good. With increasing thickness, an offset in weak reflections occurs but the linear trend is preserved, meaning various thicknesses will be amenable to the same structure solution methods.

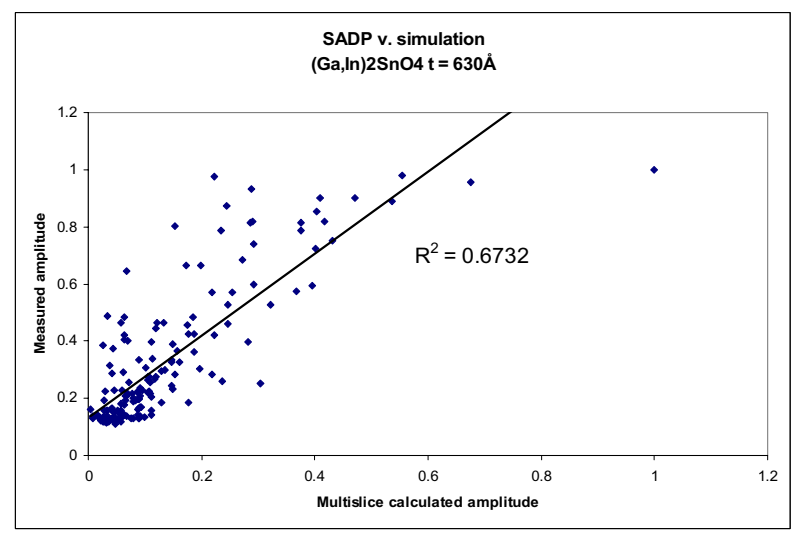

Fig. 2. Experimental SADP data v. simulation for $\mathrm{t}=630 \AA$ (same experimental conditions and specimen area as in fig. 1, excluding hollow cone illumination). This thickness yields the best match between experiment and simulation. Considerable scatter due to thickness variation is apparent. 